A N N A L E S Annales de Bretagne et des Pays de l'Ouest

Anjou. Maine. Poitou-Charente. Touraine

109-4 | 2002

Les étrangers dans l'Ouest de la France (XVIII $-\mathrm{XXX}^{\mathrm{e}}$ siècle)

\title{
Français et Américains en Basse-Seine à la Libération (1944-1946) : des relations ambivalentes
}

Marc Bergere

\section{(2) OpenEdition}

Journals

Édition électronique

URL : http://journals.openedition.org/abpo/1532

DOI : $10.4000 /$ abpo.1532

ISBN : 978-2-7535-1488-1

ISSN : 2108-6443

Éditeur

Presses universitaires de Rennes

Édition imprimée

Date de publication : 20 décembre 2002

Pagination : 203-215

ISBN : 978-2-86847-794-1

ISSN : 0399-0826

Référence électronique

Marc Bergere, «Français et Américains en Basse-Seine à la Libération (1944-1946) : des relations ambivalentes ", Annales de Bretagne et des Pays de l'Ouest [En ligne], 109-4 | 2002, mis en ligne le 20 décembre 2004, consulté le 23 avril 2019. URL : http://journals.openedition.org/abpo/1532 ; DOI : 10.4000/abpo. 1532 


\title{
Français et Américains en Basse-Seine à la Libération (1944-1946) : des relations ambivalentes
}

\author{
Marc BERGERE \\ ATER, CRHISCO - Université Rennes 2
}

En de nombreux lieux, l'arrivée des Américains constitue l'une des images fortes de la Libération. Dans bien des villes et villages, les mêmes scènes de liesse et de GI's joyeux distribuant chewing-gums et cigarettes sont ancrées dans la mémoire collective. Paradoxalement, si beaucoup de régions ont vu passer des soldats américains, plus rares sont celles où ils ont stationné durablement. À l'échelle de l'Ouest, la Seine-Inférieure représente pour cette raison un observatoire privilégié permettant de dépasser ces images emblématiques mais fugaces de la Libération. Dès lors, après avoir rapidement présenté les conditions et l'organisation de l'implantation américaine en Basse-Seine ${ }^{1}$, la problématique consiste surtout en une étude de la perception des libérateurs par l'opinion publique locale et audelà d'un certain imaginaire social des États-Unis et des Américains en France à la Libération ${ }^{2}$.

1. Sur cette dimension plus factuelle, on peut se reporter aux travaux suivants : BonDIL, Hélène, La présence américaine en Seine-Inférieure 1944-1946, mémoire de maîtrise, Université Paris 1, 1996, 131 p.; HAMEL, Ludivine, Les Américains au Havre et dans sa région 1944-1946 : réalisations et impacts, mémoire de maîtrise d'histoire, Université du Havre, 2001, 108 p. et 100 p. d'annexes; MARQUIS, Jean-Claude, Les camps " cigarette ", Rouen, Édition Médianes, 1994, 58 p.; BaCCARA, Daniel, HaRel, Jean-Michel et Moulin, Valérie, Le Havre $16^{\text {th }}$ Port of embarkation, septembre 1944-21 octobre 1946, Le Havre, Édition USST 488, 1997.

2. Si de nombreux aspects des relations franco-américaines à la Libération nous sont connus (participation aux opérations militaires, rapports politiques intergouvernementaux...), force est de constater que l'angle de l'opinion reste à ce jour peu étudié. À signaler cependant les travaux de TORRENT, Régine, "L'image du soldat américain en France de 1943 à 1945 ", Cochet, F., Genet-Delacroix, Marie-Claude et Trocme, Hélène, Les Américains et la France 1917-1947 : engagements et représentations, Maisonneuve et Larose, 1999, p. 230-243 : poursuivant sa réflexion sur le sujet, elle prépare un livre sur les Américains en France 1943-1945 sous le titre provisoire Une présence souhaitée, un accueil contrasté; BouRHIS, Philippe, Le sentiment anti-américain dans la région nantaise au lendemain des deux guerres mondiales (1918-1924/1945-1947) : étude comparée, mémoire de maîtrise d'histoire, Université de Nantes, 1987, 273 p. 


\section{Une présence américaine massive}

Les Américains ne sont pas stricto sensu les libérateurs de la région. En effet, le département de la Seine-Inférieure est libéré par les Anglo-canadiens entre le 30 août et le 12 septembre 1944, non sans avoir souffert de terribles bombardements préalables ${ }^{3}$. Malgré les destructions subies, le port du Havre est finalement retenu par l'état-major américain en quête d'une base logistique apte à soutenir le front. C'est en effet parmi les ports libérés à cette date, celui qui dispose du plus gros potentiel, notamment en matière de bassins à flot accessibles aux plus grands navires et cargos sans parler des quais, docks et autres équipements de levage. Au-delà de cet aspect technique, la proximité du front dans une zone désormais considérée comme sécurisée, ce qui n'est pas le cas d'Anvers libéré depuis le 4 septembre, plaide aussi en faveur du Havre.

\section{Des flux énormes selon un rythme ternaire}

À compter d'octobre 1944, le port du Havre devient, en terme de longévité comme d'intensité de trafic, le premier port " américain " français voire européen. Certaines sources vont même jusqu'à souligner qu'aucun port au monde n'a vu passer autant de troupes américaines au cours de cette guerre. Si l'information reste difficilement vérifiable en l'état, il n'en demeure pas moins que les flux ont été ici colossaux. Trois phases peuvent être distinguées :

- d'octobre 1944 à mai 1945 : le port du Havre est un port de débarquement de troupes et de matériels vers le front;

- de mai 1945 à septembre 1945, inversion des flux : Le Havre devient un port de réembarquement et partiellement de redéploiement des troupes vers le Pacifique;

- de septembre 1945 à l'été 1946, la fonction de réembarquement demeure mais Le Havre est désormais un port de démobilisation.

En tout, 3675000 soldats américains ont transité par le port du Havre entre octobre 1944 et août 1946. Pour la seule période octobre 1944-décembre 1945, 1355943 hommes ont été débarqués tandis que 1494123 rem-

3. Après Lorient et Saint-Nazaire en janvier-février 1943, Le Havre subit les effets dévastateurs de la stratégie du Carpet-bombing (ou tapis de bombe) chère au maréchal de l'Air Arthur Harris du Bomber command britannique, particulièrement les 5 et 6 septembre 1944. À la libération de la ville le 12 septembre, Le Havre est une cité anéantie où le bilan pour l'ensemble du conflit s'établit comme suit : 4500 immeubles partiellement détruits, 12500 immeubles totalement détruits, 21000 logements disparus sur 48000, 35000 sinistrés totaux, 65000 partiels, 5126 morts. Arch. municipales du Havre, chronologie de la reconstruction. Rouen, pour sa part, connaît 16 bombardements entre le 19 avril et le 27 août 1944. Voir aussi Amouroux, Henri, Joies et douleurs du peuple libéré 6 juin-1 ${ }^{\text {er }}$ septembre 1944, tome 8 de La grande histoire des Français sous l'Occupation, Paris, R. Laffont, p. 335-382; Huguen, Roger, "La stratégie du Bomber command britannique et son application en Bretagne 1940-1944 ", SAINCLIVIER, Jacqueline et BougEARD, Christian, La Résistance et les Français : enjeux stratégiques et environnement social, Rennes, PUR, 1995, p. 41-51. 
barquaient ${ }^{4}$. Les états mensuels révèlent des flux supérieurs à 250000 hommes par mois notamment entre mars et novembre 1945. C'est pour répondre à l'ampleur de ce trafic que le commandement américain décide d'implanter en Basse-Seine de gigantesques camps de transit. Ils s'inscrivent en complément de deux autres aires de regroupement : la Champagne avec, aux environs de Reims, 17 camps d'une capacité totale de 250000 hommes $^{5}$ et la région de Marseille avec les camps des rives de l'étang de Berre (Delta base section). À l'exception du camp Herbert Tareyton, répondant au nom d'un populaire dessinateur de pin-up, tous sont baptisés de grandes marques de cigarettes. Au nombre de huit (Home Run, Wings, Herbert Tareyton, Philip Morris, Pall Mall, Lucky Strike, Old Gold, Twenty Grand), ils disposent au cœur du pays de Caux, d'une capacité d'accueil voisine de 200000 hommes : avec ses 60000 places, Lucky Strike est le plus vaste camp de ce genre en Europe.

\section{"Les États-Unis and Caux"}

À l'exception de certaines troupes sédentaires liées à l'activité portuaire ou à la gestion des camps (la 89e division d'Infantery notamment), l'écrasante majorité des soldats américains n'effectue qu'un bref séjour (de 72 heures à 10 jours en moyenne) en Seine-Inférieure. Au-delà de ce brassage, les effectifs cantonnés n'en demeurent pas moins considérables. La présence américaine est massive et donc visible pour les populations : ils représentent en permanence près de 10 \% de la population du Havre entre 1944 et 1946. Dans les zones rurales voisines des camps, la pression démographique est parfois bien supérieure : ainsi, la population totale des six communes d'implantation du camp Lucky Strike n'atteint pas le quart de la population américaine qui y stationne ${ }^{6}$.

Rapportée aux 846131 habitants recensés en Seine-Inférieure, la population de ces « huit villes américaines ${ }^{7}$ " a été en permanence supérieure à $10 \%$

4. Sources des statistiques : US Navy et Port autonome du Havre. Arch. municipales du Havre, H4 15.5 : " History of the US Navy " in Le Havre-France, novembre 1945, 14 p.; Baccara, Daniel, Harel, Jean-Michel et Moulin, Valérie, Le Havre $16^{\text {th }}$ port..., op. cit., p. 91. Dans le même temps, 2500000 tonnes de marchandises et près de 80000 véhicules ont transité par le port du Havre entre octobre 1944 et août 1946.

5. Cette aire de regroupement était divisée en quatre secteurs : Mourmelon, Suippes, Mailly et Sissonne. Clause, Georges, "Reims autour du 7 mai 1945 ", VAISSE, Maurice, 8 mai 1945 : la victoire en Europe, Lyon, La Manufacture, 1985, p. 412-414.

6. Dans le même ordre d'idées, les 7867 habitants du ressort de la brigade territoriale de Cany-Barville côtoient au quotidien plus de 60000 Américains des camps Lucky Strike mais aussi Old Gold. Source : SHGN (Service historique de la gendarmerie nationale), BT Cany-Barville, R2, 1944-1946.

7. Immenses camps de toiles (tentes) mais aussi de baraquements en bois (type tropical wood) ou métallique (type niessen), ce sont de véritables villes "made in America" avec leur plan en damier tiré au cordeau. Les axes nord-sud sont numérotés alors que les axes ouest-est portent les noms de villes américaines (Philip Morris) ou des principaux dirigeants alliés (Herbert Tareyton). Ces villes disposent non seulement de tous les services nécessaires à la vie du camp (sanitaire, cuisine, hôpital) mais aussi de théâtre, 
de l'ensemble départemental entre 1944 et 1946. Une telle présence impose de lourdes contraintes (réquisitions, prestations en nature diverses ${ }^{8} \ldots$..), mais offre aussi des avantages indéniables (grands travaux, offres d'emploi, aides et dons multiples ${ }^{9}$ ). C'est aussi l'occasion de découvrir un autre monde...

\section{Des relations franco-américaines ambivalentes}

\section{Des relations en trompe-l'œil}

De prime abord, on peut être tenté de s'arrêter superficiellement à deux séries de comportements d'ailleurs très contrastés. D'une part, toutes les sources consultées témoignent de relations entre autorités locales américaines et françaises non seulement très satisfaisantes mais le plus souvent cordiales et dépassant le formalisme protocolaire. D'autre part, les mêmes sources révèlent aussi les inévitables frictions et crispations suscitées par la cohabitation toujours difficile entre des civils et une troupe nombreuse. De fait, la présence américaine est génératrice de multiples incidents. Les accidents de la route sont très fréquents en raison de l'intensité du trafic mais aussi du comportement de chauffeurs toujours " prioritaires ", et circulant à grande vitesse, avec peu ou pas d'égards pour les civils. De même, les très nombreuses agressions et bagarres... créent un réel climat de violence et d'insécurité au Havre et autour des camps. Un rapide comptage à travers la presse locale permet pour la seule ville du Havre de relever entre octobre 1944 et octobre 1946 : 94 accidents de la circulation, 60 agressions ou rixes et 16 décès de civils engageant la responsabilité des Américains ${ }^{10}$. Un récapitulatif des crimes et délits commis par les soldats américains, depuis leur arrivée, dans le ressort de la section de gendarmerie du Havre, enregistre au 10 août 1945 : 3 meurtres, 6 viols, 6 agressions (dont deux tentatives de viols), 28 vols (dont dix d'automobiles) et 7 bris de clôture ou violations de domicile ${ }^{11}$. Dès lors, la crainte s'installe :

cinéma, bar, terrains de sport, Px (magasins) ravitaillés en produits américains, chapelle catholique, temple protestant... Sources : Arch. municipales du Havre, H4 15.8, plan du camp Herbert Tareyton, sans date; AM de Gonfreville-l'Orcher, Du village à la ville... 19451995, film vidéo produit et réalisé par la ville, 1996, 62 mn (images du camp Philip Morris).

8. Réquisitions importantes de terres agricoles, mais aussi de logements et d'équipements collectifs particulièrement au Havre. La surconsommation d'eau et d'électricité nécessaire au ravitaillement des camps ne va pas sans poser des problèmes à la population civile dans une région sinistrée. Arch. municipales du Havre, H4 15.5, présence américaine au Havre : réquisitions, prestations diverses...

9. Au Havre, les Américains assurent la remise en état du port tout en participant aux premiers travaux de reconstruction : déblaiement de la ville, déminage, remise en état des réseaux (routiers, ferroviaires...). Plusieurs milliers de civils français travaillent à leur service (4000 dockers sur le $16^{\text {th }}$ Port en avril 1945).

10. BACCARA, Daniel, HAREL, Jean-Michel et MoULIN, Valérie, " chronologie havraise à travers l'actualité au jour le jour 12-09-44/21-11-46 ", Le Havre $16^{\text {th }}$ Port..., op. cit., p. 41-78. Si l'on considère que la presse ne répercute que les faits divers les plus graves, les chiffres issus de notre comptage ne constituent certainement qu'une évaluation plancher.

11. SHGN, carton 14340, R2 section Le Havre, 600/2, rapport du commandant de la section du Havre, le 10 août 1945. Notons que dans le même temps 33 délits ont été commis 
" Les cultivateurs s'enferment chez eux et n'osent plus sortir, les ouvriers pour ne pas passer sur les routes bordant les camps effectuent de nombreux détours pour regagner leur domicile car en plus des agressions, des coups de feu tirés assez fréquemment sans motif ne sont pas pour calmer la population ${ }^{12}$."

En Seine-Inférieure, comme dans de nombreuses régions françaises, les noirs américains sont fréquemment présentés comme les principaux fauteurs de trouble ${ }^{13}$.

À leur sujet, l'opinion locale se révèle paradoxale : surprise et heurtée par la ségrégation qui règne dans les rangs américains, elle n'en témoigne pas moins d'une grande défiance ${ }^{14}$ et parfois de préjugés racistes à l'égard des troupes noires ${ }^{15}$. Les concentrations militaires et masculines drainent par ailleurs vers la région un nombre considérable de prostituées locales ou extérieures (Paris, Nantes, Rennes...), occasionnelles ou professionnelles. La prostitution est endémique malgré l'action conjuguée des autorités américaines et françaises pour y remédier. Au-delà des inquiétudes d'ordre sanitaire ${ }^{16}$, les populations rurales du Pays de Caux notamment sont choquées par son caractère ostentatoire :

« Dans n'importe quel endroit même à la vue du public et chose plus grave à celle des enfants qui trouvent un peu partout des préservatifs et jouent avec. Tout ceci amène un état d'esprit déplorable parmi cette population agricole peu habituée à voir s'établir un genre de vie aussi scandaleuse ${ }^{17}$."

par des Français au préjudice des Américains. Sur la question des viols commis par les soldats américains en France à la Libération, voir l'étude de Robert LiLlY, à paraître dans Vingtième Siècle, Revue d'Histoire.

12. SHGN, carton 14347, R2 Section Yvetot, 767/2, rapport du commandant de la section, le 29 novembre 1945.

13. Les mêmes accusations se retrouvent en Normandie, Picardie, Moselle, Bretagne... TORRENT, Régine, op. cit., p. 238-239. Lire aussi sur cette question Louis Guilloux, O.K. Joe!, Gallimard, coll. Folio, 1976, 155 p.

14. Beaucoup de rapports font état d'une peur assez générale des troupes noires notamment au sein de la population féminine. À noter cependant que la préférence accordée aux noirs par certaines femmes est systématiquement stigmatisée par les hommes "blancs " (français ou américains).

15. À titre d'exemple, " la population ne comprend pas pourquoi en dehors de la MP, les soldats américains notamment ceux de couleur sont laissés porteurs d'armes à feu " ou encore ce rapport de la brigade de Cany-Barville : "Beaucoup de soldats américains sont stationnés dans la région. Les troupes blanches sont assez bien considérées, il n'en est pas de même des noirs qui font l'objet d'une répulsion générale de la population, provenant de leurs mœurs primitives à l'égard des femmes et pour leur intempérance notoire. "SHGN, carton 14330, R2 compagnie Seine-Inférieure, 1355/2, rapport du commandant de compagnie, le 15 octobre 1945; carton 14360, R2 brigade de Cany-Barville, 50/2, rapport du comandant de brigade, le 8 février 1945.

16. Le docteur Th. Sebald, responsable du service de médecine des femmes au Havre parle de 3000 prostituées dans la ville dont 150 à 200 hospitalisées au service de prophylaxie (contre 8 à 20 maximum avant-guerre). Arch. municipales du Havre, Havre-Éclair, PER 60, le 25 mars 1946, p. 2.

17. SHGN, carton 14347, R2 section Yvetot, 767/2, rapport du commandant de Section, le 29 novembre 1945. Au nom d'un certain hygiénisme, les Américains interdisaient la prostitution dans ou à proximité immédiate des camps. 
Les Américains sont enfin acteurs mais aussi victimes d'un intense marché noir et de trafic en tout genre (aliments, vêtements, tabac, essence...). Tous ces faits, crimes et délits fournissent à la population de nombreux et souvent réels motifs de récriminations et critiques contre les soldats américains décrits comme violents, et se comportant "en pays conquis ". Les plaintes et pétitions abondent, on voit même se constituer des groupes d'autodéfense au Havre. Sans nier la réalité de cette exaspération, il apparaît cependant qu'entre les relations officielles excellentes et les relations conflictuelles, la perception des Américains par la population française est plus nuancée et évolutive.

\section{Une relation complexe}

Deux périodes sont aisément repérables dans l'évolution de l'opinion locale à l'égard des Américains ${ }^{18}$. Dans une première phase qui s'étend de la Libération jusqu'au printemps 1945, ils sont accueillis avec enthousiasme et intérêt. Reconnaissance mais aussi attente forte d'une région meurtrie paraissent guider l'attitude des populations à l'égard de soldats dont on souligne "l'affectivité ", " la courtoisie " ou encore " la prévenance " dans le comportement. À partir de l'été et plus encore de l'automne 1945, la dégradation des rapports franco-américains est nette. Désormais, la thématique de la désillusion est déclinée sur tous les tons : le commandant de la section d'Yvetot souligne que " le français qui s'apprêtait à accueillir ses libérateurs à bras ouvert a été sérieusement douché en s'apercevant d'abord de (illisible dans le rapport) que le soldat américain lui témoignait ${ }^{19}$ ", son collègue de Rouen insiste « l'attitude des troupes américaines en particulier a fortement déçu ${ }^{20}$ ". Dès lors, certains rapports parlent d'un véritable rejet des libérateurs : en septembre 1945, le commissaire central du Havre note qu'aux yeux de l'opinion « les boches se tenaient mieux que les Américains et ne se livraient pas comme eux au vandalisme : on ne dirait pas que les Américains sont nos amis ${ }^{21}$ ". Le mois suivant, la gendarmerie de Rouen souligne à son tour :

18. Nous utilisons comme source principale les rapports de gendarmerie sur l'état d'esprit de la population. Rédigés à tous les échelons - brigade (canton), section (arrondissement), compagnie (département) - ils bénéficient pleinement d'une proximité réelle à la population notamment dans les campagnes. Souvent consignés dans les registres de correspondance confidentielle (R4), ils sont ici enregistrés dans les registres de correspondance courante (R2). Quant aux procès-verbaux des brigades voisines des camps américains, si par un effet loupe bien compréhensible, ils signalent surtout les rapports conflictuels et/ou délictueux entre civils et militaires, ils offrent cependant en seconde lecture à travers les prises de parole de gens ordinaires un auxiliaire précieux pour l'analyse de l'opinion.

19. SHGN, carton 14347, R2 section Yvetot, 669/2, rapport du commandant de la section, le 13 octobre 1945.

20. SHGN, carton 14354, R2 section de Rouen, 2328/2, rapport du commandant de la section, le 17 octobre 1945.

21. Rapport cité par MARQuIs, Jean-Claude, " La Seine-Inférieure ", Buton, Philippe et Gulllon, Jean-Marie, Les pouvoirs en France à la Libération, Belin, 1994, p. 387. 
" Notons toujours une xénophobie grandissante dans nos campagnes. Les populations ont hâte de voir partir les troupes alliées [...]. Il n'est pas rare d'entendre faire un parallèle entre la tenue des troupes allemandes d'occupation et les troupes américaines ${ }^{22}$."

Les Renseignements généraux de la région rémoise, elle aussi confrontée à une concentration importante de troupes américaines constatent la même dégradation selon une chronologie identique. Là aussi, après une période de cordialité, on est passé à une période d'incompréhension et de tensions. À compter du printemps 1945, le sentiment général marnais à l'égard des Américains est devenu mauvais voire parfois " haineux ${ }^{23}$ ". Dans les deux cas, la coïncidence avec la victoire finale est frappante. Le décrochage de l'opinion correspond à la victoire militaire en Europe et plus encore sur tous les fronts. La présence américaine acceptée puis tolérée jusque-là devient insupportable dès lors qu'elle n'est plus nécessaire à la protection. Comme si le prolongement des contraintes inhérentes au temps de guerre devenait trop pesant après le retour à la paix. Yves-Henri Nouailhat avait déjà constaté cette rupture à l'égard des Américains à Saint-Nazaire entre 1917 et 1919 : la signature de l'armistice ouvrant le temps du désenchantement. On relève d'ailleurs la même impatience au sujet de la prolongation des difficultés de la vie quotidienne et au sortir de l'hiver 1944-1945, les lendemains qui déchantent n'épargnent plus personne pas même les nouvelles autorités françaises ou les forces neuves issues de la Résistance. La désaffection qui frappe les Américains, si elle peut être ressentie comme une forme d'ingratitude, mérite donc d'être replacée dans un mouvement plus général d'opinion de populations polarisées par les problèmes matériels et de plus en plus divisées sur des questions telles que l'épuration, les élections, l'école ou encore la constitution ${ }^{24}$. Pour autant, les troupes américaines ont peut-être une part de responsabilité. En effet, il est incontestable que l'inversion des flux (débarquement vers le front - rembarquement pour redéploiement ou démobilisation), qui s'opère au printemps 1945, s'accompagne d'un net relâchement de la discipline. Plusieurs rapports s'en font l'écho et de fait les incidents civils/militaires explosent au second semestre 1945. Pour la seule ville du Havre, on dénombre entre le $1^{\mathrm{er}}$ janvier et le $1^{\mathrm{er}}$ juin 1945,9 accidents, 6 agressions et un décès de civil imputables aux Américains alors que la deuxième partie de l'année livre 42 accidents, 43 agressions ou rixes et 8 civils tués. Dans un tel climat, la lassitude des populations paraît légitime. En ce sens, la hâte à voir partir les Américains est une réaction d'irritation

22. SHGN, carton 14354, R2 section de Rouen, 2328/2, rapport du commandant de la section, le 17 octobre 1945.

23. Lire Husson, Jean-Pierre, " Le retentissement de la victoire de la Marne " et CAUSE, Georges, « Reims autour du 7 mai 1945 », VAISSE, Maurice, 8 mai 1945..., op. cit., p. 375-378, 390-393 et 399.

24. Dans l'ensemble du pays, la thématique d'une population peu compréhensive et se repliant de plus en plus sur un égoïsme régional, départemental ou communal est très présente dans les rapports d'opinion à compter du printemps 1945. 
et de peur compréhensible face à des troupes nombreuses dont certains éléments se comportent mal. Ce réflexe que l'on retrouve souvent au sein de tout territoire soumis à une forte densité militaire non choisie (et ce quelle que soit la nationalité de l'armée) relève davantage de l'épidermique et du conjoncturel que du sentiment profond. D'ailleurs, la presse locale tout en répercutant les faits et les plaintes se garde bien de tout amalgame hâtif, le journal Normandie préférant titrer :

"Quelques dizaines de mauvais garçons comme on en trouve sous toutes les latitudes et sous tous les uniformes vont-ils suffire à discréditer l'armée d'une nation à laquelle nous unissent tant de souvenirs fraternels ${ }^{25}$ ? "

De la même manière, il faudrait pouvoir opérer le distinguo entre les troupes sédentaires en général mieux intégrées et les troupes de passage, par tradition, plus remuantes et incontrôlées. Au-delà de ces réserves nécessaires, il n'en demeure pas moins que l'opinion normande nous livre des éléments, plus profonds et spécifiques à la relation franco-américaine. Ainsi, le rejet des Américains qui semble se généraliser en 1945 n'a jamais fait cesser l'admiration qu'ils suscitent. Au contraire, les deux sentiments coexistent en permanence et se nourrissent l'un de l'autre. Les Américains ont été à terme dans cette région (comme ailleurs vraisemblablement) victimes des qualités qu'on leur prêtait ou plutôt qu'on attendait d'eux. En effet, la fascination pour la puissance américaine est réelle et omniprésente dans les sources consultées. Les Havrais sont particulièrement impressionnés par la puissance militaire des États-Unis, mais aussi par leur armada mécanique (jeeps, bulldozers, scrapers, engins motorisés en tout genre...) et leur richesse économique. Tout paraît extraordinaire chez eux, leur équipement, leurs vêtements, les produits qu'ils consomment, leur organisation sans faille. Perçus sous cet angle, les camps américains sont de véritables "villes champignons " qui poussaient " en quelques heures, en quelques jours tout au plus, avec l'ampleur, les méthodes et la vitesse qui sont de règle outre-atlantique ${ }^{26}$ ". Avec eux, tout va vite : ils construisent vite, ils conduisent vite... Derrière ces exagérations qui font des ÉtatsUnis un eldorado où tout est plus facile, un véritable pays de Cocagne pour eux et pour les autres, se cache une perception stéréotypée classique véhiculée dès cette époque par la bande dessinée ou le cinéma ${ }^{27}$. Représentation excessive sans doute mais qui n'en demeure pas moins vecteur d'opinion. À ce stade, il n'est d'ailleurs pas exagéré d'affirmer que la population normande a été victime du " mirage américain ${ }^{28}$ " : elle attendait beaucoup des libérateurs, de leur puissance, de leur richesse et plus encore de leur capacité réelle ou supposée à peser sur le quotidien d'où

25. Arch. dép. de Seine-Maritime, 2 MI 1484 R1, journal Normandie, 9 octobre 1945, p. 2. 26. Jean Le Povremoyne dans le journal Normandie du 22 janvier 1946. Article cité dans MARQUIS, Jean-Claude, Les camps “ cigarette ", op. cit., p. 18.

27. À titre d'exemple, cette représentation des États-Unis, pays de la rapidité et de l'efficacité optimale se retrouve aussi bien dans Tintin en Amérique (Hergé, 1931), que dans Jour de fête (Jacques Tati, 1949).

28. Velllon, Dominique, Vivre et survivre en France 1939-1947, 1995, Payot, p. 304-307. 
l'ampleur de la frustration. Ici, l'incompréhension est à la mesure de l'admiration que l'Amérique et ses hommes provoquaient. Dès lors que les difficultés quotidiennes persistent voire parfois s'aggravent, les Américains constituent des boucs-émissaires tout désignés. D’ailleurs, selon un sondage cité par Jean-Pierre Rioux, 55 \% des Français considèrent, dès janvier 1945, l'aide américaine comme insuffisante ${ }^{29}$.

Quelques mois plus tard, à la question " depuis un an, quel(s) pays a(ont) déçu vos espérances? ", les États-Unis devancent largement la Grande-Bretagne et plus encore l'URSS ${ }^{30}$. Le désaveu est à la hauteur des espoirs, sans doute démesurés, placés dans l'intervention américaine, les mêmes sondages révélant qu'à la Libération, l'Amérique était l'allié dont les Français attendaient le plus. Sur ce désamour, ne tardent pas à se greffer d'autres griefs. De manière plus ou moins diffuse, l'opinion selon laquelle les Américains sont un frein à notre propre redressement tend à se répandre. Dans son rapport mensuel, l'officier commandant la section de gendarmerie d'Yvetot note en août 1945 : " La population française a la nette impression que les alliés mettent obstacle à notre redressement ${ }^{31}$. " Ce même mois, dans un éditorial du journal Normandie, on peut lire :

"Affaiblie, ayant du mal à retrouver son équilibre, consciente cependant de son passé et de ses forces futures, la France souffre de tout ce qui paraît atteindre sa fierté et son intégrité nationale. Or, nous avons un peu l'impression que les États-Unis glorieux à juste titre de leurs jeunes, victorieuses et sensationnelles forces, croient apporter au monde un nouvel évangile. Et ils l'apportent avec une certitude d'eux-mêmes qui parfois nous déconcerte ${ }^{32}$."

Paradoxalement, les autorités américaines partagent en partie ce point de vue. Le très francophile général de brigade Bryan Conrad, chargé par l'ambassade américaine à Paris d'analyser l'hostilité croissante entre civils français et GI's, conclut en août 1945 son rapport sur l'idée que

« les Français sont blessés, instables, ils souffrent d'un complexe d'infériorité collectif [...]. La France aujourd'hui est malade, affamée, fière et difficile à manœuvrer ${ }^{33}$."

29. Rioux, Jean-Pierre, La France de la Quatrième République. L'ardeur et la nécessité 1944-1952, Paris, Le Seuil, coll. Points Histoire, 1980, p. 19 (Bulletin d'information de l'IFOP, janvier 1945).

$30.54 \%$ des sondés se disent déçus des États-Unis contre $12 \%$ seulement pour l'URSS. A contrario, ne sont pas déçus par les États-Unis $38 \%$, par la Grande-Bretagne $43 \%$ et par I'URSS $71 \%$. (Bulletin d'information de l'IFOP, juillet 1945). Résultats cités dans Rioux, Jean-Pierre, "Cette immense joie pleine de larmes : les Français et le jour V ", VAISSE, Maurice, 8 mai 1945 ..., op. cit., p. 333-334; RUPNIK, Jacques et LACORNE, Denis, « La France saisie par l'Amérique ", LACORNE, Denis, RUPNIK, Jacques et TOINET, Marie-France, s.d., L'Amérique dans les têtes, Paris, Hachette, 1986, p. 25.

31. SHGN, carton 14347, R2 section Yvetot, 642/2, rapport du commandant de la section, le 11 août 1945.

32. Arch. dép. de Seine-Maritime, 2 MI 1484 R1, journal Normandie, 25 et 26 août 1945.

33. CONRAD, G. Bryan, "Franco-American Relations, 14 août 1945 ", cité par Frank Costigliola, " L'image de la France aux États-Unis", mages et imaginaires dans les relations internationales depuis 1938, Les Cahiers de l'IHTP, 1994, n 28 (s.d. Robert FranK), p. 98. 
En effet, par un jeu de miroir classique, on peut se demander jusqu'à quel point l'image positive des Américains ne renvoyait pas de manière trop crue une image négative de la France. À parcourir la presse normande, on devine que la puissance nord-américaine est d'autant plus mal vécue qu'elle révèle aux Français leur propre faiblesse. Dans Le Havre-Éclair du 11 juin 1945, Pierre Aubéry, de retour d'une visite au camp Philip Morris, lâche :

"Une activité jamais ralentie règne partout. Des camions circulent, les bulldozers manœuvrent. Inlassablement, des grues coopèrent à l'érection de nouveaux poteaux télégraphiques, les scrapers nivellent le sol. C'est une ville complète qui s'élève à côté du Havre, avec une existence propre, son personnel, ses commodités, ses plaisirs aussi. On se demande ce que pense le paysan qui, à quelques mètres du camp, conduit sa charrue minuscule, trâ̂née par un unique cheval. Auprès des monstres mécaniques qui pétaradent à l'envie, autour de nous, il paraît bien désuet, bien désarmé, ne retarde-t-il pas un peu ${ }^{34}$ ?"

Plus explicite est cet autre article du 9 juillet 1945 :

"On ne peut se défendre d'un sentiment d'envie lorqu'on voit passer, rapides, sinon élégantes les jeeps innombrables de l'armée US. Quatre pneus neufs, plus bien souvent, un de rechange, 25 ou 30 litres d'essence aux 100 kilomètres, voilà des caractéristiques qui ne trompent pas et nous font mieux mesurer notre misère. Pour rouler ainsi, il faut avoir derrière soi un pays riche, de pétrole, de gomme, toute une organisation industrielle qui nous fait défaut ${ }^{35}$. "

L'admiration désabusée dont témoigne ce journaliste se mue parfois en réaction de colère et de honte face aux sollicitations permanentes dont sont l'objet les soldats américains. Pour de nombreux observateurs, cette volonté de grappiller " leurs miettes " offre une image pénible et déshonorante de la France ${ }^{36}$. Dans son rapport mensuel d'octobre 1944, la gendarmerie de Rouen signale « la mendicité est à tel point générale et outrancière qu'un soldat allié avait écrit à la craie sur son camion "no cigarettes pour papa" 37 ". En avril 1945, c'est à la presse havraise de s'élever à son tour contre de telles pratiques :

"Ce spectacle écœurant, trop fréquent ici et ailleurs : une nuée de gosses de tous âges, piaillant, tirant les soldats par la manche, demandant à longueur de journée : "cigarettes pour papa" ou bien "chewing-gum for me please" [...]. Quelle opinion veut-on que les soldats américains rapportent dans leur pays après la guerre d'une France qui se tient aussi mal? Hélas, on le devine aisément et le bon garçonnisme des Américains blancs ou noirs n'y change rien, il y a du mépris dans leur sourire ${ }^{38}$."

34. Article cité dans MARQuis, Jean-Claude, Les camps " cigarette », op. cit., p. 43.

35. "Chronologie havraise à travers l'actualité au jour le jour ", op. cit., p. 57.

36. En Normandie mais aussi dans le Nord ou le Loiret, cf. VEILLON, Dominique, Vivre et survivre..., op. cit., p. 306.

37. SHGN, carton 14352, R2 section de Rouen, 228/2, rapport du commandant de la section, le 15 octobre 1944.

38. "Chronique havraise à travers l'actualité au jour le jour ", op. cit., p. 50-51. 
Comme souvent, l'envie se transforme en critique et on en vient rapidement à en vouloir aux Américains d'étaler tant d'aisance et de richesse dans une France en pénurie. Dès lors, à la puissance et à l'abondance dont ils témoignent, l'opinion accole souvent le sans-gêne, le gaspillage voire la provocation. Dans un autre registre, les Américains apparaissent d'autant plus suspects aux yeux des normands qu'ils témoignent d'une trop grande bienveillance aux Allemands. Au-delà des litiges diplomatiques qui se jouent au même moment autour de la question allemande, c'est le dossier des prisonniers de guerre (PW) qui cristallise localement les tensions. Pour de nombreux havrais, ces derniers sont trop bien traités, trop libres et trop nourris ${ }^{39}$. Les autorités américaines ont beau opposer par voie de presse la convention de Genève et démentir les rumeurs les plus folles qui circulent, leur sympathie pour " le boche " est fréquemment stigmatisée.

Même s'ils s'en défendent, leur mansuétude à l'égard des PW allemands est ressentie comme une préférence accordée à l'ennemi et tend à faire de l'antigermanisme un vecteur d'antiaméricanisme ${ }^{40}$. Comme après la Grande Guerre, on redoute confusément que le sort réservé aux PW ne soit le prélude d'une politique américaine germanophile privant par la même occasion la France des fruits de "sa " victoire et d'une juste revanche. À cette époque, les Français doutent d'ailleurs massivement de la fermeté américaine. Répondant à la question " quels sont les peuples qui seront partisans de traiter assez durement l'Allemagne? ", 87 \% pensent aux Russes, $48 \%$ aux Britanniques et seulement $9 \%$ aux Américains ${ }^{41}$.

Ambivalentes, les relations franco-américaines révèlent finalement toutes les ambiguïtés et la complexité d'une opinion construite en grande partie sur des représentations. Sans négliger l'importance de la chronologie (déroulement des opérations militaires), des rapports entre le politique et le culturel (influence de la question allemande) ou du climat spécifique généré par une présence militaire massive, il faut ici souligner l'impact de l'image qu'on se construit de l'ailleurs avant même de le connaître. Ainsi, les Américains, idéalisés de longue date au même titre que la Libération tant attendue, ont déçu lorsqu'ils se sont révélés incapables de satisfaire l'espoir placé en eux. Effet miroir aussi d'une image trop positive qui rend plus

39. La presse locale qui reçoit sur ce point un abondant courrier de lecteurs s'en fait largement l'écho notamment la presse communiste (l'Avenir du Havre en particulier). Il existe plusieurs camps de prisonniers de guerre allemands dans la région (à Bolbec notamment) et les Américains emploient nombre d'entre eux à leur service (transport, administration...).

40. Philippe Bourhis observe le même phénomène dans la région de Nantes-SaintNazaire, Le sentiment antiaméricain dans la région nantaise..., op. cit. Les autorités américaines sont d'ailleurs pleinement conscientes du caractère central de cette question dans l'antagonisme franco-américain à la libération. Leurs enquêtes sur les principaux griefs formulés par les GI's à l'encontre des Français révèlent de fait une relative faveur à l'Allemagne, Costigliola, Frank, "L'image de la France aux États-Unis ", op. cit., p. 99.

41. Bulletin d'information de l'IFOP, 16 juillet 1945 cité par Rioux, Jean-Pierre, "Cette immense joie pleine de larmes... " dans 8 mai $1945 \ldots$, op. cit., p. 324. 
criante encore les faiblesses de la France d'alors. Comme souvent, le regard porté sur l'Autre est aussi un regard sur soi. Réciproquement, les malentendus franco-américains sont aussi nés d'une vision stéréotypée des Français par les Américains ${ }^{42}$. Le journaliste américain Joe Weston, dans un long article, démontre parfaitement combien ses concitoyens ont débarqué en France avec des idées préconçues dans leur paquetage ${ }^{43}$. La plupart voyait la France comme un pays arriéré, sale, peuplé de femmes faciles et d'hommes lâches car vaincus. Tous s'attendaient aussi à " se faire arnaquer ". Conscient que "Le Havre fut la première ville française à ressentir pendant un temps non négligeable [cet] esprit GI's " et à en subir " les pires conséquences ", il reconnaît aussi que beaucoup de Havrais avaient une vision des États-Unis « sans grand rapport avec la réalité ». Il précise :

"Ils s'attendaient à voir des gens, aimables, riches, instruits, individualistes, des sentimentaux au cœur d'or pratiquant cette démocratie dont nous faisons tant l'éloge. En lieu et place de quoi, ils se sont trouvés face à de soidisant "démocrates" qui détestaient les noirs [...]. Ils ont vu "des sentimentaux au cœur d'or" qui distribuaient de la nourriture et des friandises aux seules femmes affamées qui pouvaient les payer. Ils ont rencontré des "individualistes purs et durs" qui traitaient la population locale comme des êtres inférieurs. Ils ont vu des Américains "aimables et romantiques" accoster tout ce qui portait une jupe."

Autocritique, il regrette " cette incompréhension que ni les autorités françaises, ni l'armée américaine n'ont véritablement cherché à dissiper " au point " qu'elle est devenue un cancer qui ronge la France [...], le coup de grâce [ayant été] asséné par la propagande allemande que les GI's ont avalé sans broncher et qui prétendait que les Allemands étaient gentils et propres et les Français horribles ". Ce qui se joue au Havre à la Libération, c'est finalement la rencontre voire l'affrontement de deux prétentions à la puissance dont l'une est d'autant plus éclatante que l'autre est exsangue et n'a pas ou plus les moyens de sa politique. Comme souvent dans les relations entre les deux pays ${ }^{44}$, Américains et Français forment un couple repoussoir qui mêlent en permanence admiration et irritation, fascination et humiliation.

42. Cf. Costigliola, Frank, op. cit.

43. Article publié dans le magazine Life du 31 décembre 1945, p. 32 sous le titre : "Les GI's au Havre : les soldats américains venus combattre en France, loin d'être acclamés comme des libérateurs, sont considérés comme des émissaires malveillants ". Joe Weston introduit l'article par cet avertissement : "Ceci est l'article le plus difficile qu'il m'ait été donné d'écrire en tant que journaliste. Pendant près de quatre ans, j'ai été soldat dans l'armée américaine. Je rapporte ici l'histoire d'hommes avec lesquels j'ai combattu et de camarades qui sont morts. Je ne veux porter aucun jugement sur mes compatriotes. J'accuse toutefois les acteurs du système éducatif américain, les parents et ceux qui font les films de propagande, de vendre une Amérique qui n'existe pas. De le vendre non seulement au monde mais ce qui est pire encore, à l'Amérique elle-même. "

44. L'ambivalence constitue une tendance forte des relations franco-américaines. Pour une mise en perspective sur un temps plus long, lire notamment les contributions d'André KASPI, Jacques RuPNIK ou André LACORNE, dans l'Amérique dans les têtes, op. cit. 


\section{RESUME}

Point majeur d'entrée mais aussi de sortie du territoire, le grand Ouest entretient un rapport privilégié aux Américains durant les deux conflits mondiaux. Situation unique à l'échelle française voire européenne, le port du Havre et la Basse-Seine constituent, à compter d'octobre 1944, un énorme centre de transit pour les troupes américaines : les " camps cigarettes " au nom de marques célèbres (Pall Mall, Lucky Strike, Philip Morris...) ont accueilli plusieurs centaines de milliers d'Américains entre 1944 et 1946. Face à ces flux massifs et durables, quelles ont été les réactions des populations locales? Après avoir situé la présence américaine en Seine-Inférieure (SeineMaritime aujourd'hui), cette communication démontre surtout le caractère ambivalent des relations franco-américaines témoignant au passage du poids des représentations dans les phénomènes d'opinion.

\section{ABSTRACT}

As a major passageway in and out of the French territory, the Western regions of France maintained a privileged relationship with the Americans during two major world conflicts. Unique situation on a French and even European scale, the port of Le Havre and the Basse-Seine region became an enormous transit zone for American troops as from october 1944: the so called "cigarette camps", named after famous American brands of cigarettes accomodated hundreds of thousands of American people between 1944 and 1946. Faced with such constant flows of people entering and leaving France, how did the local population react?

Having situated the American presence in the Seine-Inferieure region (now called Seine-Maritime), this two way communication symbolises above all the ambivalent nature of French-American relationships, thus revealing the impact on people's minds. 
